Jochen Bauer*, Hilko Hoffmann, Ingo Zinnikus, Thomas Feld, Mathias Runge, Oliver Hinz, Michael Hechtel, Christoph Konrad, Martin Holzwarth, Andreas Mayr, Sven Schneider, and Jörg Franke

\title{
ForeSight - Al-based Smart Living Platform Approach
}

https://doi.org/10.1515/cdbme-2020-3099

\begin{abstract}
In the upcoming years, the internet of things (IoT) will enrich daily life. The combination of artificial intelligence (AI) and highly interoperable systems will bring contextsensitive multi-domain services to reality. This paper describes a concept for an AI-based smart living platform with open$\mathrm{HAB}$, a smart home middleware, and Web of Things (WoT) as key components of our approach. The platform concept considers different stakeholders, i.e. the housing industry, service providers, and tenants. These activities are part of the ForeSight project, an AI-driven, context-sensitive smart living platform.
\end{abstract}

Keywords: smart home, smart living, web of things, interoperability

\section{Motivation}

The economic relevance of connected homes or buildings is proven by several key figures $[1,2]$. German Federal Minister Peter Altmaier described the market for smart living applications as a „mega-ecosystem of the future“, with ,annual growth rates of 25-30\%" and expected revenues in Germany of ,tens of billions by 2025“ [3]. The size of the market is relevant, i.e. if all 43 million households by 2030 were equipped with smart home technology with an average value of 3,000 EUR, this would result in a market potential of 129 billion EUR [4].

\footnotetext{
*Corresponding author: Jochen Bauer, Michael Hechtel, Christoph Konrad, Martin Holzwarth, Andreas Mayr, Sven Schneider, Jörg Franke, Institute for Factory Automation and Production Systems, Fürther Straße 246b, 90429, Nuremberg, Germany, e-mail: jochen.bauer@faps.fau.de

Hilko Hoffmann, Ingo Zinnikus, Deutsches Forschungszentrum für Künstliche Intelligenz GmbH, Stuhlsatzenhausweg 3, 66123 Saarbrücken, Germany

Thomas Feld, Strategion GmbH, Albert-Einstein-Straße 1, 49076 Osnabrück, Germany

Mathias Runge, loT connctd $\mathrm{GmbH}$, Hardenbergstraße 32 , 10623 Berlin, Germany

Oliver Hinz, Faculty of Economics and Business Administration, Goethe University Frankfurt, Theodor-W.-Adorno-Platz 4, 60323 Frankfurt am Main, Germany
}

There are several definitions of the terms smart home and smart living, that range from cell phone controlled lights to full-fledged agent-controlled systems of all devices in several buildings [5]. Common relevant domains for smart home are energy, health, and home automation [6]. We define the term smart home as a group of more than three devices which are able to be controlled by another device and offer useful information to the user. In addition, smart living integrates use cases that are not limited to the location of the local home network.

Most smart home systems offer their users a central access point in form of a user interface, for example, a smartphone app or an installed display. Usually, available system devices are supplied by the manufacturer and an integration of foreign components, i.e. from different ecosystems, is normally unwanted. The open-source middleware openHAB offers an abstraction layer for devices and services. The core of the openHAB middleware uses the layers 5 to 7 of the OSI model. There are several addons or bindings, which address other layers of the OSI model as well, e.g. the network binding works on layer 4. Moreover, there are vendor- and protocolspecific bindings, called to communicate with devices and services. This is the lowest level of interoperable systems, socalled technical interoperability. One step further is to agree on specific syntax or file formats, like CSV or XML for data storage or data exchange, known as syntactic interoperability. Regarding the IoT, it is useful to strive for the next higher level of interoperability, the semantic interoperability. At this level, machines are able to detect the meaning from the data transfer, i.e. it is possible for the machine to understand what task needs to be completed to fulfill the specific use case. As a result, the machine can develop its own optimization strategies [7]. The availability and interchangeability of information about who is allowed to do what kind of actions lead to the next level of interoperability, the organizational or pragmatic interoperability. Such a degree of interoperability has not been achieved in the smart living sector yet. Besides, there are more levels of interoperability, which are not considered in this paper. To get an impression of requirements it is helpful to analyze system approaches related to the smart living reference architecture model [8].

To include semantic information into systems, ontologies can be used. These are semantic orders of terms related to a do- 
main and their relationships [9]. One of these ontologies is the WoT [10], which uses so-called Thing Descriptions [11] and represents a standard recommended by the $\mathrm{W} 3 \mathrm{C}$ to connect (IoT) objects to the web. There are several research projects which are using WoT-based approaches for the smart living domain. In addition to symbolic AI methods like using ontologies, data-driven machine learning (ML) tools are gaining more and more attention. Last but not least, these days lots of innovative US located companies are offering their cloud platforms for storing all this data, so a possibility for a holistic cloud abstraction layer, which follows German and European privacy and security requirements as well as improved data sovereignty, will be helpful for European companies. Summing up, both ontology- and ML-based approaches are relevant in the smart living domain and need to be chosen wisely to solve upcoming challenges as well as an overall integrated identity and access management.

In the following section, we will identify current challenges, which need to be addressed to utilize the WoT with openHAB in the context of an AI-based smart living platform. Afterwards, we provide a concept for an approach with respect to the mentioned challenges, which will be discussed at the end of the paper.

\section{Challenges}

Recently, middleware applications are beginning to ensure interoperability [12]. The capabilities of visual-based configuration tools for smart home environments are improving, plug and play, comfortable device discovery, and installation procedures are more and more available. Remote access is possible by an additional cloud service, which is usually provided by the system vendor or are run on premise. More and more systems offer comfortable tools to configure the devices of the smart home and the corresponding UI. Summing up, the costs for a smart home are much lower than five years ago, but there is still a need for improving the interoperability between the systems to increase the benefits for the tenants. Furthermore, systems are offering application programming interfaces (API) access to third party applications like IFTTT, and so the user is able to automate procedures on his own without having much expertise in software development. Anyway, the possibility to bring existing business models to an IoT-based ecosystem needs to be improved. In a first step, a promising platform approach needs to offer interoperability through all layers of the ecosystem. In a second step, tools for configuring business models into digital services, which will use the platform, need to be created.
Ontologies should be considered by almost any smart living platform project due to their ability to be extended. These approaches should be combined with ML and an appropriate strategy to store data. Nevertheless, it needs to be considered which side effects are occurring due to the lack of existing tools for the work with AI, i.e. relevant and existing ontologies. Smart home systems neither consider the complete lifecycle of a building nor relevant processes of the housing industry's business cases. These stakeholders and their specific requirements need to addressed as well; otherwise, the targeted economic impact of the ForeSight platform will be unlikely. Increasing the availability of semantic information is a necessary first step for enabling further improvements of the current smart living ecosystems.

\section{Approach}

In our ForeSight approach, smart home devices can be controlled by openHAB or other smart home middleware systems like digitalSTROM. WoT will be used as a mechanism to store vendor-independent data models. On top of WoT, providers are offering their services, i.e. smart home wide infotainment services. To minimize the effort to offer such services, these companies need to have access to tools, which are helping to create these services from companies' business models, i.e. create a service like an IFTTT action with the help of a UI-based configuration dialogue.

In ForeSight, AI plays an important role to achieve different goals, i.e. for offering so-called base services for energy management, object identification, localization as well as identity and access management. Sometimes it is necessary to use a cloud-based infrastructure to perform calculations, occasionally it is important that the relevant data will not leave the local network, so AI-based operations need to be done on embedded or edge devices to ensure tenants' privacy regards. Privacy and security issues requirements are key factors for a successful platform concept. Therefore, we extended the current openHAB software to take these requirements into account [8]. Furthermore, data storage concepts need to address these requirements as well, i.e. the current Gaia- $X$ initiative, which is driven by Germany and Europe to address the challenge of an hyperscaler-independent abstraction layer with a focus on data sovereignty [14].

ForeSight uses a layer-based architecture to enable horizontal and vertical interoperability, thus reducing almost all vendor- or company-specific dependencies (see Fig. 1). Horizontal interoperability ensures the cross-domain and manufacturer compatibility of devices. In contrast, vertical interoperability provides interchangeability between multiple services, 
e.g. use cases where services are used by clients, which are probably tenants from different energy suppliers. In ForeSight we strive to create service engineering tools, so that existing businesses will be able to master the digital transformation of their business models effortlessly.

Due to the mentioned challenges above, IoT devices or groups of such devices need to be as smart as possible. In the concept of thinking objects (TOs), several strands of research are combined: smart environments, i.e. the physical infrastructure (sensors, actuators, and networks), ambient intelligence which refers to a ,digital environment that proactively, but sensibly, supports people in their daily lives“ [13], and AI, in particular agent systems and a variety of ML techniques. TOs represent singular physical as well as software-defined devices, temporary or stable aggregations, and combinations of physical and virtual devices. TOs aggregate and abstract sensor data of devices. Abstraction and aggregation are also a prerequisite for the provision of value-added services to users instead of (raw) data and information. TOs, which represent sensors and actuators in a room or the lighting in a building, can be combined for coordinated activities, e.g. to create a pleasant atmosphere or to guide the residents through the building. The coordination can be controlled by a predefined behavior or a self-organized, goal-directed activity. To achieve this, TOs need to tackle several challenges. They need to integrate themselves in highly connected environments, but they should offer their services in barely connected and automated surroundings, too. A seamless connection can be achieved by means of syntactic and semantic interoperability. Conceptually, TOs are an extension of multi-agent systems, which provide a flexible paradigm for an integral framework, relating the different properties such as reactivity (acting in a timely fashion), autonomy, proactiveness (taking the initiative), and communicative social behavior [15]. Agents perceive the environment through sensors, acquire new correlations based on the perceptions, and possess reasoning abilities to select and execute actions. Devices and software entities need to be coordinated in an intelligent environment. A wide range of techniques for behavioral modeling, rule-, planning-, and logicbased approaches can be used for coordinating actions. A critical factor is the collection of contextual information such as location, time, temperature, and, more challenging, the intention of the user. TOs must learn to intervene only when necessary or when they are asked to do so. Activity learning [16] is the key to reliable context detection. Accordingly, devices in the ForeSight ecosystem act as TOs.

After describing the layer-based ForeSight architecture and the idea of TOs, we focus on the connection of the middleware, here openHAB, as one of ForeSight's middleware systems and WoT to add semantic information. An openHAB addon has been developed to transform openHAB things into the
WebThing object model utilizing the Mozilla WebThings Java framework. The WebThings are hosted on a WebThing server to which the addon can connect to by using an API. Status changes of the items are communicated using websockets and REST API calls. Services can also use this interface to interact with the objects through the web. Each thing provides a thing description which can be used by humans and machines to interpret the semantics, and thus capabilities of the object (see Fig. 2) are known to everyone. The combination of aggregating the data of all devices and the existing semantic data history in the cloud makes it possible to create a digital twin of the smart living ecosystem with all corresponding benefits.

\section{Discussion}

Due to the utilization of the WoT architecture defined by the W3C, the smart home objects will be made available in a data model that was designed with cross-domain interoperability in mind. Therefore, the services of the ForeSight platform will be able to consume relevant information easily, and application development for horizontally and vertically integrated software will have a low barrier of entry for business partners. Additionally, the ForeSight consortium consists of multiple industry partners who are experienced in running IoT platforms. Therefore, it seems promising to find a team of companies which will run the platform in the future and thus eliminate potential questions regarding the future of the platform after the research project has ended.

\section{Author Statement}

Research funding: This work was supported by the BMWi (ForeSight). Conflict of interest: Authors state no conflict of interest.

\section{References}

[1] IDCWorldwide. Quarterly Smart

Home Device Tracker. (2019).

https://www.idc.com/getdoc.jsp?containerld=prUS44971219 (last accessed 2020-07-02)

[2] MarketsandMarkets. Smart Home Market by Product Global Forecast to 2024. (2019). https://www.marketsandmarkets.com/Market-Reports/smarthomes-and-assisted-living-advanced-technologie-and-globalmarket-121.html (last accessed 2020-07-02)

[3] Altmaier P. Zukunftsmarkt Smart Living. Symposium Smarte Wohnung von GdW, ANGA, Wirtschaftsinitiative Smart Living. Berlin (2019). https://anga.de/smarte-wohnung (last accessed 2020-07-02)

[4] ZVEI. Zukunftsmarkt Smart Living. Smart Home Haushalte in Deutschland. Frankfurt; 2017. 


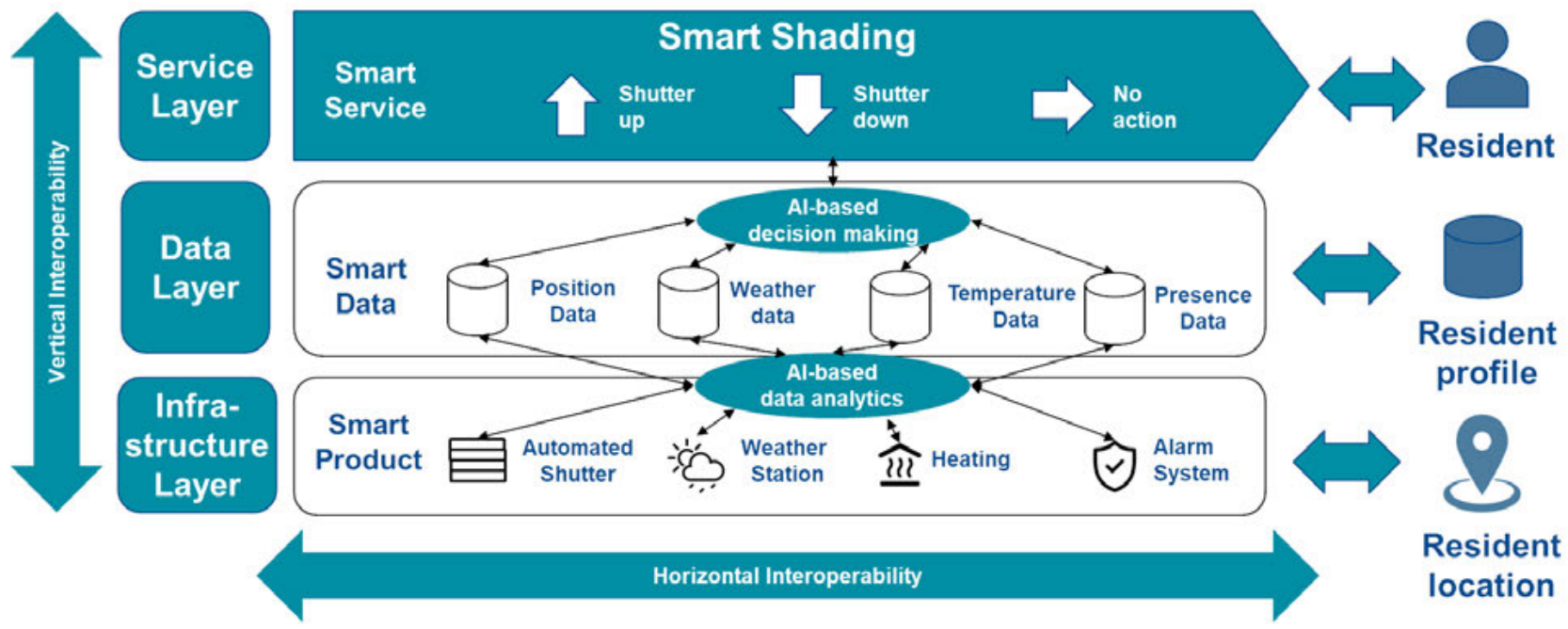

Fig. 1: ForeSight's layer-based architecture to enable horizontal and vertical interoperability for reducing almost all vendor- or companyspecific dependencies, here shown by the example of a smart shading use case.

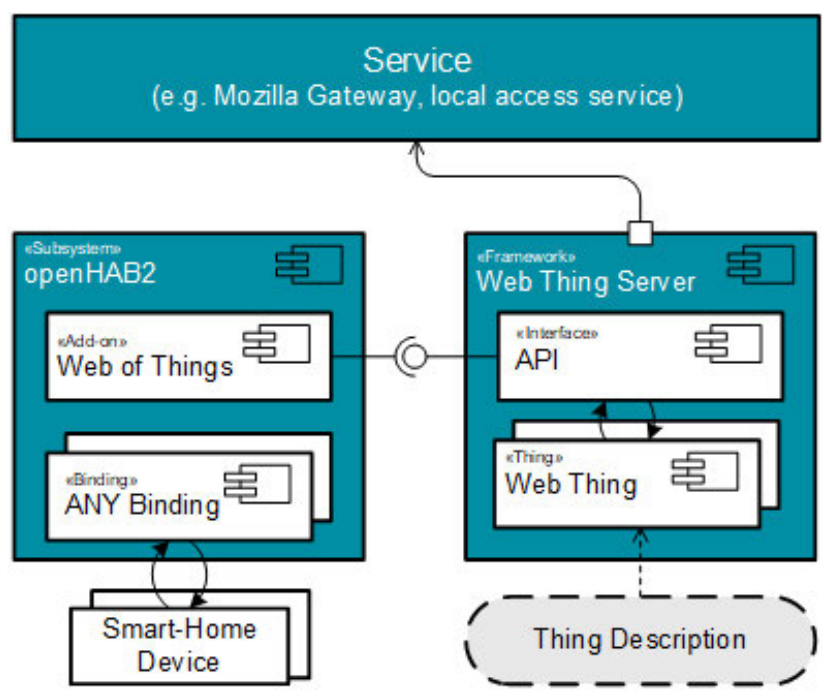

Fig. 2: ForeSight platform approach considering different smart home middleware systems, here openHAB, which interacts with a WoT technology stack. On top of these technologies companies create and connect their services as providers.

[5] M. Schiefer. Smart Home Definition and Security Threats. In: 2015 Ninth International Conference on IT Security Incident Management \& IT Forensics; 2015, p. 114-118.

[6] Bauer J, Kettschau A-K, Michl M, Bürner J, Franke J. Die intelligente Wohnung als Baustein im Internet der Dinge: Potenzialanalyse und Konzept einer domänenübergreifenden Lösung. In: Weidner R RT, editor. Erste Transdisziplinäre Konferenz zum Thema Technische Unterstützungssysteme, die die Menschen wirklich wollen. Hamburg; 2014, p. 298-307.

[7] Noura M, Atiquzzaman M, Gaedke M. Interoperability in Internet of Things: Taxonomies and Open Challenges. Mobile Netw Appl 2019;24(3):796-809
[8] Bauer J, Hechtel M, Konrad C, Holzwarth M, Hoffmann H, Feld T et al. ForeSight - An Al-driven Smart Living Platform, Approach to Add Access Control to openHAB. In: Jmaiel M, Mokhtari M, Abdulrazak B, Aloulou H, Kallel S, editors. The impact of digital technologies on public health in developed and developing countries: 18th International Conference, ICOST 2020, Hammamet, Tunisia, June 24-26, 2020, Proceedings. Cham: Springer; 2020, p. 432-440

[9] Gruber TR. A translation approach to portable ontology specifications. Knowledge Acquisition 1993;5(2):199-220.

[10] Kovatsch M, Matsukura R. Web of Things (WoT) Architecture. (2020) https://www.w3.org/TR/wot-architecture/ (last accessed 2020-07-02)

[11] Charpenay V, Käbisch S, Kosch H. Introducing Thing Descriptions and Interactions: An Ontology for the Web of Things. In: SR+SWIT@ISWC; 2016, p. 55-66

[12] Broring A, Schmid S, Schindhelm C-K, Khelil A, Kabisch S, Kramer D et al. Enabling loT Ecosystems through Platform Interoperability. IEEE Softw. 2017;34(1):54-61.

[13] Augusto JC. Ambient Intelligence: The Confluence of Ubiquitous/Pervasive Computing and Artificial Intelligence. In: Schuster AJ, editor. Intelligent Computing Everywhere. London: Springer-Verlag London Limited; 2007, p. 213-234.

[14] Hoppe G, et al. GAIA-X: A Pitch Towards Europe Statusbericht zu Anwenderökosystemen und -anforderungen. BMWi. (2020) https://www.bmwi.de/Redaktion/DE/Publikationen/DigitaleWelt/gaia-X-a-pitch-towards-europe.pdf (last accessed 202007-01)

[15] Wooldridge M, Jennings NR. Intelligent agents: theory and practice. The Knowledge Engineering Review 1995;10(2):115-52.

[16] Cook DJ. Activity learning: Discovering, recognizing, and predicting human behavior from sensor data. Hoboken, $\mathrm{NJ}$ : Wiley; 2015 